\title{
The Therapeutic Effect of ICAM-1- Overexpressing Mesenchymal Stem Cells on Acute Graft-Versus-Host Disease
}

\author{
Bo Tanga,b Xue Lia Yuanlin Liu ${ }^{a} \quad$ Xiuhui Chen ${ }^{a} \quad$ Ximei Li ${ }^{a} \quad$ Yanan Chu ${ }^{a}$ \\ Heng Zhu ${ }^{a}$ Weijiang Liu ${ }^{a, d}$ Fenfen $X^{a}$ Fan Zhou ${ }^{c}$ Yi Zhang ${ }^{a}$
}

aDepartment of Cell Biology, Institute of Military Cognitive and Brain Sciences, Academy of Military Medical Sciences, Academy of Military Sciences, Beijing, bepartment of Hematology, Peking University First Hospital, Beijing, 'The General Hospital of Shenyang Military, Shenyang, dAnhui Medical University, Hefei, China

\section{Key Words}

Mesenchymal stem cells - Intercellular adhesion molecule-1 - Graft-versus-host disease • Bone marrow transplantation • Murine GVHD model

\begin{abstract}
Background/Aims: Mesenchymal stem cells (MSCs) do not readily migrate to appropriate sites, and this creates a major obstacle for their use in the treatment of graft-versus-host disease (GVHD). Intercellular adhesion molecule-1 (ICAM-1) can guide the homing of various immune cells to the proper anatomical location within secondary lymphoid organs (SLOs), which are the major niches for generating immune responses or tolerance. MSCs rarely migrate to SLOs after intravenous infusion, and are constitutively low expression of ICAM-1. So in our previous work, ICAM- 1 was engineered into a murine MSC line $\mathrm{C} 3 \mathrm{H} 10 \mathrm{~T} 1 / 2$ by retrovirus transfection system (ICAM-1MSCs). Here, we hypothesized that ICAM-1 ${ }^{\text {high } M S C s}$ may significantly improve their immunomodulatory effect. Methods: We used different co-culture methods combined with real-time PCR and flow cytometry to evaluate ICAM- $1^{\text {high }}$ MSCs immunomodulatory effect on dendritic cells (DCs) and T cells in vitro and in vivo. MSCs were labeled with carboxyfluorescein diacetate succinimidylester (CFSE) to detect its distribution in mouse model. Results: Our in vitro analyses revealed ICAM-1 MSCs could suppress DCs maturation according to co-culture methods and suppress the $T$ cell immune response according to the mixed lymphocyte response (MLR) and lymphoblast transformation test (LTT) tests. We found that infusion of ICAM- ${ }^{\text {high }}$ MSCs potently prolonged the survival of GVHD mouse model. The infused ICAM-

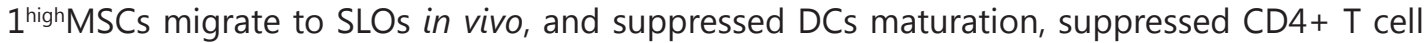
differentiation to Th1 cells, and increased the ratios of Treg cells. Conclusions: Taken together, these data demonstrate that ICAM- $1^{\text {high }}$ MSCs had an enhanced immunosuppressive effect on DCs and T cells, which may help explain the protective effect in a GVHD model. This exciting therapeutic strategy may improve the clinical efficacy of MSC-based therapy for GVHD.

B. Tang and X. Li contributed equally to this work.

Yi Zhang

and Fan Zhou

Department of Cell Biology, Institute of Basic Medical Sciences, Academy of Military

Medical Sciences Department of Hematology, The General Hospital of Shenyang

Military, Shenyang (China); E-Mail zhangyi612@hotmail.com, zhoufan611@sina.com.cn
\end{abstract}

KARGER 


\section{Introduction}

Acute graft-versus-host disease (GVHD) is a major obstacle following allogeneic hematopoietic stem-cell transplantation (HSCT) [1], which is caused by alloreactive T cells that recognize alloantigens initially presented by host/donor antigen-presenting cells (APCs) [2-4]. Although GVHD can sometimes be constrained with rigorously T cell-depleted donor grafts or pharmacologic agents, such treatments predispose patients to relapses in malignancy and opportunistic infections. Mesenchymal stem cells (MSCs) are a heterogeneous population of plastic-adherent cells with the capacity to differentiate in vitro into osteoblasts, adipocytes and chondroblasts, which have a wide range of immunosuppressive effects on innate and adaptive immune cells [5, 6]. Nearly all immune cells, including T, B, natural killer (NK), and dendritic cells (DCs), are affected. Because of their relatively convenient isolation, ex vivo expansion, and low toxicity, the clinical utility of MSCs has rapidly expanded in recent years.

However, the immunosuppressive capacity of MSCs is not always as effective as originally hypothesized. Some researchers have claimed that MSCs have a protective effect against GVHD [7]. This effect has not been observed consistently in mouse models [8]. In addition, the results of clinical trials with MSCs are also confusing, as earlier trials showed substantial benefits, whereas two recent multicenter, prospective phase III trials with at least one source of MSCs did not show any benefit $[9,10]$. Currently, it is recognized that the inefficient homing of systemically infused MSCs is a major barrier of existing MSC-based clinical GVHD therapy $[11,12]$.

Intercellular adhesion molecule-1 (ICAM-1) is a highly glycosylated protein that belongs to the immunoglobulin superfamily of cell adhesion molecules (CAMs), which were reported to play crucial roles in the specific and effective immune response to foreign pathogens [13, 14]. ICAM-1 is normally constitutively expressed on the surface of a wide variety of cell types, including leukocytes, endothelial cells, and fibroblasts [15]. It can guide the homing of various immune cells to the proper anatomical location within secondary lymphoid organs (SLOs). Normally, MSCs constitutively express a low level of ICAM-1 [16-18]. In our previous work, a recombinant retrovirus vector containing the mouse ICAM-1 gene was successful constructed [19]. The overexpressing-ICAM-1 ${ }^{\mathrm{HIGH}}$ MSCS cell line (ICAM-1MSCs) was obtained by the transfection of the MSC cell line C3H10T 1/2. Forced expression of ICAM-1 almost did not alter the immunophenotype. ICAM- $1^{\text {high }}$ MSCs are positive for CD29, CD44, and Sac-1 and are negative for CD45, CD11b, CD31 and CD86. As for the multilineage differentiation potential, ICAM- $1^{\text {high }}$ MSCs have the capacity to differentiate in vitro into osteoblasts, adipocytes and chondroblasts. Although other researchers have demonstrated that increased ICAM-1 is critical for MSC-mediated immunosuppression, the role of ICAM-1 in preventing GVHD has not been widely studied.

In this article, we show that overexpression of ICAM-1 enhances the immunomodulatory activity of MSCs and that they interact with DCs or T cells by making MSCs more adhesive to cells, which plays an important role in MSC-mediated immunosuppression. Therefore, this article uncovers a novel role of adhesion molecules in preventing GVHD in a mouse model, which will provide a potential future approach for the prevention or treatment of GVHD in patients.

\section{Materials and Methods}

\section{Mice}

Female C57BL/6 (B6, H2b) and BALB/c (BALB/c, H-2d) mice were purchased from Vital River (Charles River China). The age of the mice ranged from 8 to 10 weeks. All mice were kept under specific pathogen-free conditions and utilized in the animal center of the Academy of Military Medical Sciences. All experiments in this study were performed in accordance with the Academy of Military Medical Sciences Guide for Laboratory Animals (IACUC of AMMS-2013-021). 


\section{Cell preparations}

Bone marrow cells (BMCs) were collected from the backbones and femurs. The spleens were crushed through 40- $\mu \mathrm{m}$ screens, and then, cell suspensions were prepared by centrifugation with a Ficoll gradient at 1000x g for $20 \mathrm{~min}$ to separate leukocytes from the cell debris. The leukocytes were collected from the interface and washed before use. Primary MSCs (pMSCs) were isolated from newborn C57BL/6 murine compact bone and culture-expanded in $\alpha$-minimal essential medium ( $\alpha$-MEM, Gibco) containing $10 \%$ fetal bovine serum in a humidified atmosphere of $5 \% \mathrm{CO}_{2}$ at $37^{\circ} \mathrm{C}$. The murine MSC line C3H10T1/2 (CCL-226) was obtained from ATCC. MSCs/MSCV (C3H10T1/2 cells transfected with the empty vector), and ICAM1 MSCs were obtained as described in our previous report and passaged and expanded in MSC culture medium.

Preparation of DCs from bone marrow cells and DCs co-culture with MSCs in vitro.

BMCs from mouse ( 8 weeks old) femur were prepared by the depletion of red cells and were then cultured at a density of $2 \times 10^{6}$ cells $/ \mathrm{ml}$ in 6-well plates in minimal essential medium (MEM, Gibco, Grand Island) with $10 \%$ fetal bovine serum (FBS), $10 \mathrm{ng} / \mathrm{ml}$ of recombinant mouse granulocyte-monocyte colonystimulating factor (mGM-CSF, RD) and $1 \mathrm{ng} / \mathrm{ml}$ of recombinant mouse IL-4 (mIL-4, RD). On day 4, DCs were collected and co-cultured with pMSCs, MSCs/MSCV, or ICAM-1 MSCs at a ratio of 5:1 for 3 additional days in the presence of $1 \mu \mathrm{g} / \mathrm{ml}$ LPS with the intent of stimulating DCs maturation. At day 7, the cell morphology of the

DCs and MSCs co-cultured DCs was observed under the microscope (Nikon TE2000-U), and the DCs were collected and CD11c, CD80, CD86 and Ia were used as indicators of fully mature DCs by flow cytometry.

Mixed lymphocyte response (MLR) and lymphoblast transformation test (LTT)

As for MLR testing, MSCs or ICAM-1 MSCs were preplaced in 96-well plates at $1 \times 10^{4} / 100 \mu \mathrm{l} /$ well. The responder splenocytes from $B A L B / c$ mice $\left(2 \times 10^{5} /\right.$ well $)$ were mixed with irritating stimulator splenocytes from C57BL/6 mice (15 Gy irradiated) at various densities $\left(2 \times 10^{5}, 4 \times 10^{4}, 8 \times 10^{3} /\right.$ well). Then, these cells were added and cocultured with MSCs or ICAM-1 MSCs to obtain a final volume of $300 \mu$ l. Positive control wells contained responding and allogenetic irritating cells. As for the LTT, MSCs or ICAM-1 MSCs were preplaced in 96-well plates at various densities $\left(1 \times 10^{4}, 2 \times 10^{4}\right.$ and $4 \times 10^{4} /$ well $)$ and irradiated 24 hours later at 15 Gy. Splenic T lymphocytes from BALB/c mice were added to the coculture with MSCs at a density of $2 \times 10^{5} /$ well in 96-well plates. Concanavalin A (5 mg/ml) (ConA; Sigma Aldrich Co.) were added to promote mitogen proliferation, and a negative control well contained only splenocytes from BALB/c mice. The plates were maintained at $37^{\circ} \mathrm{C}$ for 56 hours and then pulsed with $1 \mathrm{mCi}$ of ${ }^{3} \mathrm{H}$ thymidine deoxyribonucleoside per milliliter for another 16 hours. The cells were then harvested onto glass fiber filters, and their radioactivity was measured on a Wallac Microbeta Trilux 1450-02P. All experiments were performed in triplicate and repeated three times.

\section{Murine GVHD Model}

Mice underwent transplantation according to following protocol. Briefly, the recipients received a single dose of 800 cGy total body irradiation (TBI, $\mathrm{Co}^{60} \gamma$ source). In the GVHD group, BMCs $\left(10 \times 10^{6}\right)$ and Spleen mononuclear cells (SPMNC) $\left(10 \times 10^{6}\right)$ from C57BL/ 6 mice were suspended in a total volume of 0.2 $\mathrm{ml}$ of PBS and injected intravenously into recipient animals on day 0 . pMSCs $\left(5 \times 10^{5}\right)$, MSCs/MSCV $\left(5 \times 10^{5}\right)$, or ICAM-1MSCs $\left(5 \times 10^{5}\right)$ were injected into GVHD mice 24 hours after transplantation. The body weights and survival were monitored daily, and the GVHD clinical scores were measured weekly. The degree of systemic acute GVHD was assessed using a scoring system that incorporates five clinical parameters: weight loss, posture (hunching), activity, fur texture and skin integrity. Histopathology of GVHD was monitored according to Kaplan et al. [20]. Specimens from at least three recipient mice per group were collected for experiments except where otherwise described.

In vivo tracking of exogenously infused MSCs

To track MSCs in the GVHD models, MSCs $\left(5 \times 10^{5}\right)$ were labeled with carboxyfluorescein diacetate succinimidylester (CFSE, Life Technologies) to detect the distribution of the transplanted pMSCs, MSCs/ MSCV, or ICAM-1 MSCs in vivo and then injected into the lateral tail vein of GVHD mice in a total volume of 0.2 $\mathrm{ml}$ of PBS. Forty-eight hours later, samples of the spleen, lung, liver, and intestine from the recipients were 


\section{Cellular Physiology and Biochemistry}

Cell Physiol Biochem 2018:46:2624-2635

\begin{tabular}{l|l}
\hline DOI: $10.1159 / 000489689$ & (C) 2018 The Author(s). Published by S. Karger AG, Basel
\end{tabular}

Publisned onlıne: MVay 14, 2018 www.karger.com/cpb

Tang et al.: ICAM-1highMSCs on aGVHD

collected for cryosectioning. Fluorescent cells in the sections were visualized under a fluorescence microscope equipped with a Nikon Eclipse 80i. For quantification of the CFSE + cells, the average number of green fluorescent cells per 5 scope in triplicate mice was counted at $200 \times$ magnification.

Table 1. Primer sequences

\begin{tabular}{|c|c|c|}
\hline Genes & Primer sequences & Annealing temperature \\
\hline \multirow[t]{2}{*}{ HPRT } & forward, $5^{\prime}$ - AGTCAAGGGCATATCCAACAACAA - $3^{\prime}$ & \\
\hline & reverse, $\quad 5^{\prime}$ - GCTGGTGAAAAGGACCTCTCG - $3^{\prime}$ & \\
\hline \multirow[t]{2}{*}{ TNF- $\alpha$} & forward, $\quad 5^{\prime}$-CACTTGGTGGTTTGCTACGA-3' & \\
\hline & reverse, $\quad 5^{\prime}$-GCCTCCСТCTCATCAGTTCTA-3' & \\
\hline \multirow[t]{2}{*}{ IL-4 } & forward, $\quad 5^{\prime}$ - ACTCCATGCTTGAAGAAGAACTC $-3^{\prime}$ & \\
\hline & reverse, $\quad 5^{\prime}$ - ATGATGCTCTTTAGGCTTTCCA - $3^{\prime}$ & \\
\hline \multirow[t]{2}{*}{ IFN- $\gamma$} & forward, 5'- TTACTACCTTCTTCAGCAACAGCAA -3' & \\
\hline & reverse, $\quad 5^{\prime}$ - CTGGTGGACCACTCGGATGA - $3^{\prime}$ & $60{ }^{\circ} \mathrm{C}$ \\
\hline \multirow[t]{2}{*}{ Foxp3 } & forward, $5^{\prime}$ - AGAGCCCTCACAACCAGCTA - $3^{\prime}$ & \\
\hline & reverse, $\quad 5^{\prime}$ - CCAGATGTTGTGGGTGAGTG - $3^{\prime}$ & \\
\hline
\end{tabular}

Immune factor

determination by real-time PCR and ELISA

RNase-free plastic and water were used throughout the assay. Total RNA was extracted with TRIzol reagent (Invitrogen) and reverse transcribed using the RevertAid First Strand cDNA Synthesis Kit (Thermo). cDNAs were amplified by qRT-PCR using a SYBR Green PCR kit (Sigma). The qRT-PCR results were normalized using mouse HPRT as the housekeeping gene. All cDNA sequences were obtained from the GenBank database. The primer sequences used for the qRT-PCR are listed in Table 1. Meanwhile, Mouse IFN- $\gamma$ and IL-10 concentrations were determined according to the reagent protocols of the quantitative determination kit (eBioscience).

\section{Flow cytometry analysis}

Antibodies, including PE-CY5 anti-mouse CD11c, PE anti-mouse CD86, FITC anti-mouse CD80, PE anti-mouse CDIA, FITC anti-mouse H2Kb, APC/Cy7 anti-mouse CD3, PerCP/Cy5.5 anti-mouse CD8, PE antimouse IFN-gamma, PE anti-mouse IL4, and PE/Cy7 anti-mouse IL17A, were purchased from Biolegend. The procedure was performed as described elsewhere. Briefly, for surface staining, approximately one million cells were incubated with saturating concentrations of the antibody mixture at $4{ }^{\circ} \mathrm{C}$ for 30 minutes as illustrated in the instructions. For intracellular cytokine analysis, leukocytes isolated from spleen cells were stimulated with a Cell Stimulation Cocktail with protein transport inhibitors (500×, eBioscience), which could be directly added to the culture medium at $1 \mathrm{X}(2 \mu \mathrm{L} / \mathrm{mL})$, for $4-18 \mathrm{~h}$. For intracellular cytokine staining, cells were fixed and permeabilized using the BD IntraSure Kit.

\section{Statistical analysis}

The Kaplan-Meier product-limit method was used to calculate the survival curve. Differences between groups in the survival studies were determined using log-rank statistics. For all other data, Student's t test was used to analyze differences between groups, and the results were considered significant if the $\mathrm{p}$ value was less than or equal to 0.05 . Unless otherwise specified, all error bars in this study are the S.E.M.

\section{Results}

Overexpressing ICAM-1 enhanced the suppression capacity of MSCs for DCs in vitro

DCs are specialized antigen-presenting cells that are pivotal in immune responses. It is generally accepted that immature DCs (iDC) in the periphery become mature and migrate to secondary lymphoid organs, where T cells are activated [21]. To investigate the enhanced expression of ICAM-1 on MSCs in the determination of DC fate, first, we found that numerous mature DCs (maDC) were generated from BMC using mIL4/mGM-CSF supplemented with an additional LPS incubation (Fig. 1 B). In terms of the cellular morphology, maDC were found to be relatively disperse and had long synapses (Fig. 1 A). After 3 days of co-culture with MSCs, numerous DCs were adhered to MSCs, and the long synapses could not be seen under a microscope, especially for ICAM-1 MSCs (Fig. 1A). Surface expression of costimulatory 
Fig. 1. ICAM-1 $1^{\text {high }}$ MSCs suppressed the maturation and differentiation of DCs in vitro. (A) Morphologic characteristics of DCs: iDC (a); maDCs with longer synapses (b); DCs co-cultured with pMSCs (c); DCs co-cultured with MSCs (d); DCs co-cultured with MSCs/MSCV (e); DCs co-cultured with ICAM- $1^{\text {high }}$ MSCs(f). After 3 days of coculture with MSCs, numerous DCs adhered to MSCs, and long synapses could not been seen under a microscope, especially in ICAM- $1^{\text {high }}$ MSCs (f). (B) Phenotypic characteristics of DCs: expression of CD80, CD86 and Ia gated on CD11c were decreased by MSCs. The data are shown from one representative experiment of three replicates. ${ }^{*}, \mathrm{p}<0.05$; ${ }^{* *}$, $\mathrm{p}<0.01$; ***, $\mathrm{p}<0.001$, n.s. not significant. Error bars indicate SEM). Abbreviations: iDC, immature DCs; maDC, mature DCs; pMSCs, primary MSCs; MSCs, a murine MSC line

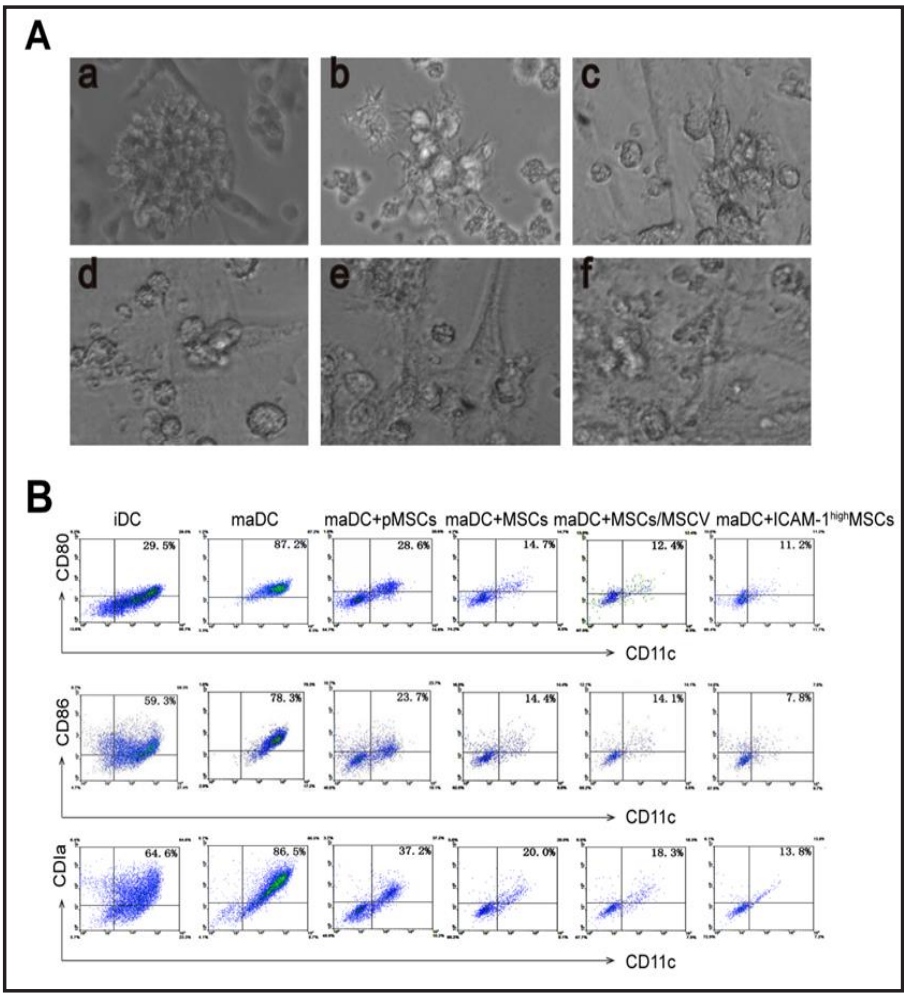
C3H10T1/2 cells; MSCs/MSCV, a murine MSC line C3H10T1/2 cells transfected with the empty vector; ICAM- $1^{\text {high }}$ MSCs, overexpressing-ICAM-1 MSC cell line.

molecules and MHC II is critical for the function of DCs. We compared the expression of CD80, CD86 and Ia on DCs by flow cytometry. The expression of CD80, CD86 and Ia decreased in DCs co-cultured with MSCs, especially in ICAM$1^{\text {high }}$ MSCs (Fig. 1B).

O verexpressing ICAM-1 enhanced the immunosuppression capacity of MSCs

Next, studies were performed to assess the in vitro suppressive capacity of MSCs in MLR and LTT cultures. The results showed that pMSCs, MSCs, MSCs/MSCV, and ICAM$1^{\text {high }}$ MSCs all inhibited the proliferation of lymphocytes in vitro in a cell dose-dependent manner. Splenocytes co-cultured with ICAM-1 $1^{\text {high } M S C s}$ had the lowest proliferative response compared with the MSC/MSCV group ( $p<0.05$; Fig. 2).

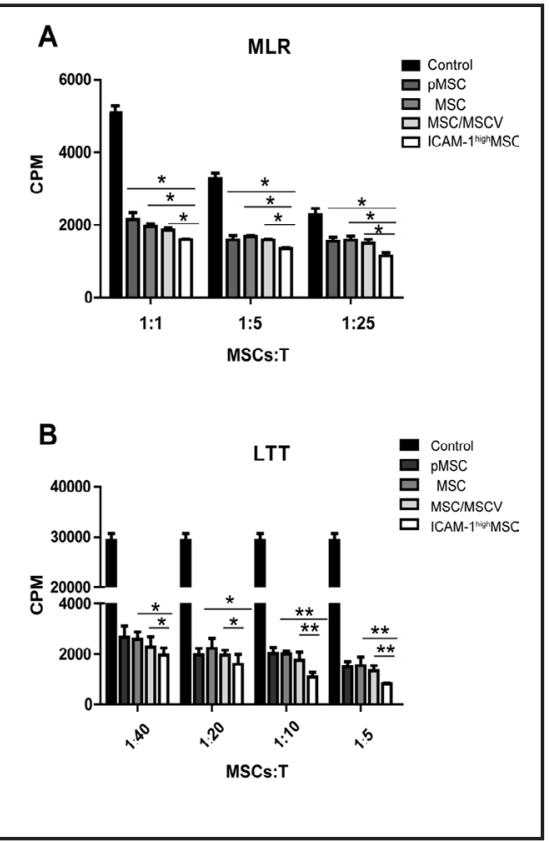

Fig. 2. Enhanced expression of ICAM-1 enhanced the immunomodulatory capacity of MSCs in vitro. Various doses of MSCs were added to the MLR and LTT system, and T-cell proliferation was analyzed via $3 \mathrm{H}$ thymidine uptake. The data are shown from one representative experiment of three replicates. $\left(^{*}, \mathrm{p}<0.05\right.$; $* *, \quad \mathrm{p}<0.01 ; * * *, \mathrm{p}<0.001$, n.s. not significant. Error bars indicate SEM). Abbreviations: pMSCs, primary MSCs; MSCs/MSCV, a murine MSC line C3H10T1/2 cells transfected with the empty vector; ICAM-1 $1^{\text {high }}$ MSCs, overexpressingICAM-1 MSC cell line. 
Fig. 3. ICAM-1 high $^{\text {MSCs }}$ remarkably inhibits the development of lethal GVHD. (A) Survival curves of each group of mice $(n=15)$. (B) Degree of the systemic GVHD scores of the GVHD, pMSCs, MSCs/MSCV and ICAM-1 ${ }^{\text {high }}$ MSCs groups of mice. (C) Paraffin sections of liver, intestine and lung of three animals/treatment group were analyzed for histological signs of GVHD on the day mice were killed due to their moribund state or at the end of the experiment. The data are shown from one representative experiment of three replicates ${ }^{*}, \mathrm{p}<0.05 ; * *, \mathrm{p}<0.01$; ***, $\mathrm{p}<0.001$, compared with the pMSCs or MSC/ MSCV infusion group). Abbreviations: GVHD, graft-versus-host disease; pMSCs, primary MSCs; MSCs/MSCV,

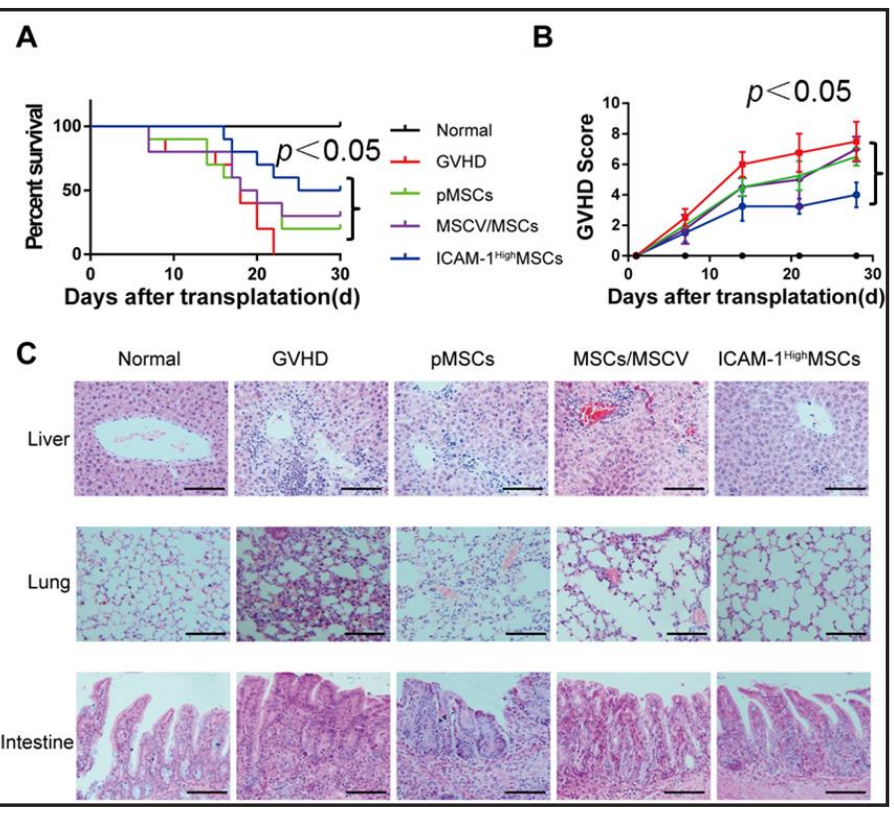
a murine MSC line C3H10T1/2 cells transfected with the empty vector; ICAM-1 $1^{\text {high }}$ MSCs, overexpressing-ICAM-1 MSC cell line.

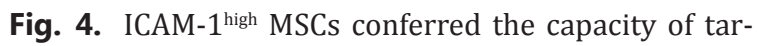
geted migration to secondary lymphoid organs in vivo. (A) pMSCs, MSCs/MSCV and ICAM- $1^{\text {high }}$ MSCs were labeled with the green fluorescent dye CFSE and were then intravenously injected into GVHD mice. Five days later, the organ sections containing cells labeled with CFSE were observed by fluorescence microscopy (green staining). DAPI counterstaining was performed to distinguish recipient cells (blue staining). Bar $=100 \mu \mathrm{m}$. (B): Quantification of the CFSE+ cells: the average number of green fluorescent cells per 5 scope in triplicate mice was counted at $200 \times$ magnification. The data are representative of three independent experiments. The data represent the mean value \pm SD of at least 5 mice $\left(*, p<0.05\right.$; ${ }^{* *}$, $\mathrm{p}<0.01 ;{ }^{* * *}, \mathrm{p}<0.001$, n.s. not significant. Error bars indicate SEM, compared with the MSCs or MSC/MSCV infusion group). Abbreviations: GVHD, graft-versus-host disease; pMSCs, primary MSCs; MSCs/MSCV, a murine MSC line $\mathrm{C} 3 \mathrm{H} 10 \mathrm{~T} 1 / 2$ cells transfected with the empty vector; ICAM- $1^{\text {high }}$ MSCs, overexpressing-ICAM-1 MSC cell line; CFSE, carboxyfluorescein diacetate succinimidyl ester.

Enforced expression of ICAM-1 on MSCS significantly ameliorated the severity of

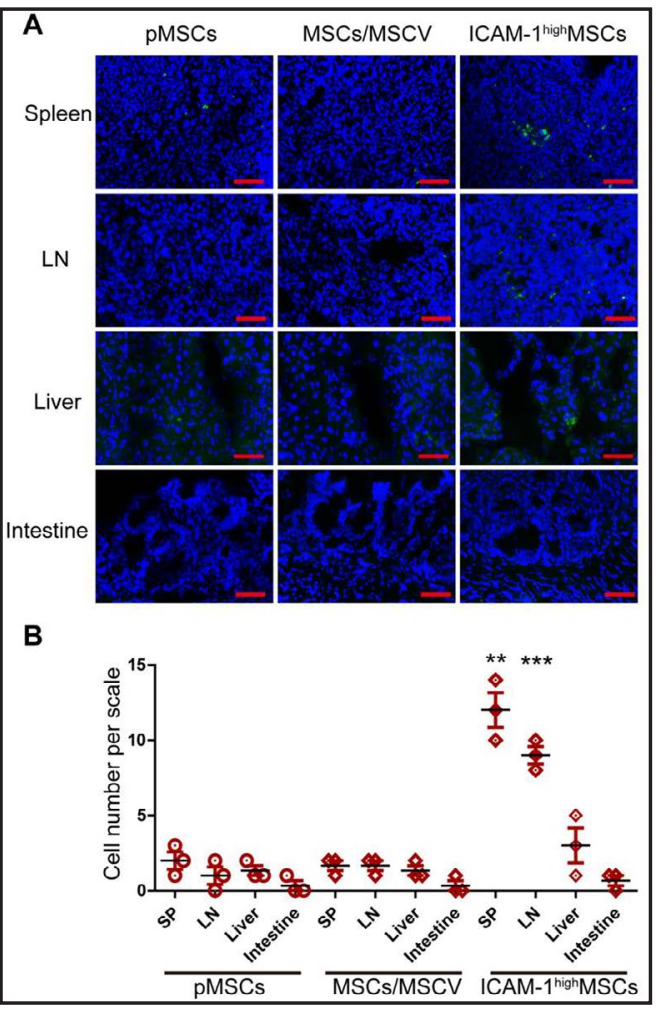
acute GVHD

Since ICAM-1 MSCs gained enhanced adhesion and suppression abilities toward DCs and T cells in vitro, we hypothesized that overexpression of ICAM-1 on MSCs could improve their immunosuppressive activity in vivo. We tested this hypothesis in a murine GVHD model. The transplantation of allogeneic BM and spleen cells induced severe clinical GVHD with high GVHD scores and a lethality of $100 \%$ within 25 days after transplantation. The injection 
Fig. 5. ICAM- $1^{\text {high }}$ MSCs suppressed the maturation, differentiation and function of DCs in vivo. Seven days after ICAM- $1^{\text {high }}$ MSC treatment, spleens of the model mice were harvested. (A) Phenotypic characteristics of DCs: expression of CD11c, CD80 and CD86 was detected by FCAS. ICAM- $1^{\text {high }}$ MSCs down-regulated the percentage of cells positive for CD11c, CD80 and CD86. (B) The difference in percentage of CD11c, CD80 and CD86 was analyzed. The data are the mean of 5 independent experiments and a representative result is shown. The data represent the mean value \pm SD of at least 5 mice $(*, p<0.05 ; * *, p<0.01$; ***, $\mathrm{p}<0.001$ ). Abbreviations: DCs, dendritic cells; GVHD, graft-versus-host disease; pMSCs, primary MSCs; MSCs/MSCV, a murine MSC line C3H10T1/2 cells transfected with the empty vector; ICAM-1 $1^{\text {high }}$ MSCs, overexpressing-ICAM-1 MSC cell line.

of pMSCs reduce mortality to 20\% (Fig. $3 A$ ), while ICAM-1 MSCs infusion could rescue mortality to approximately 50\%

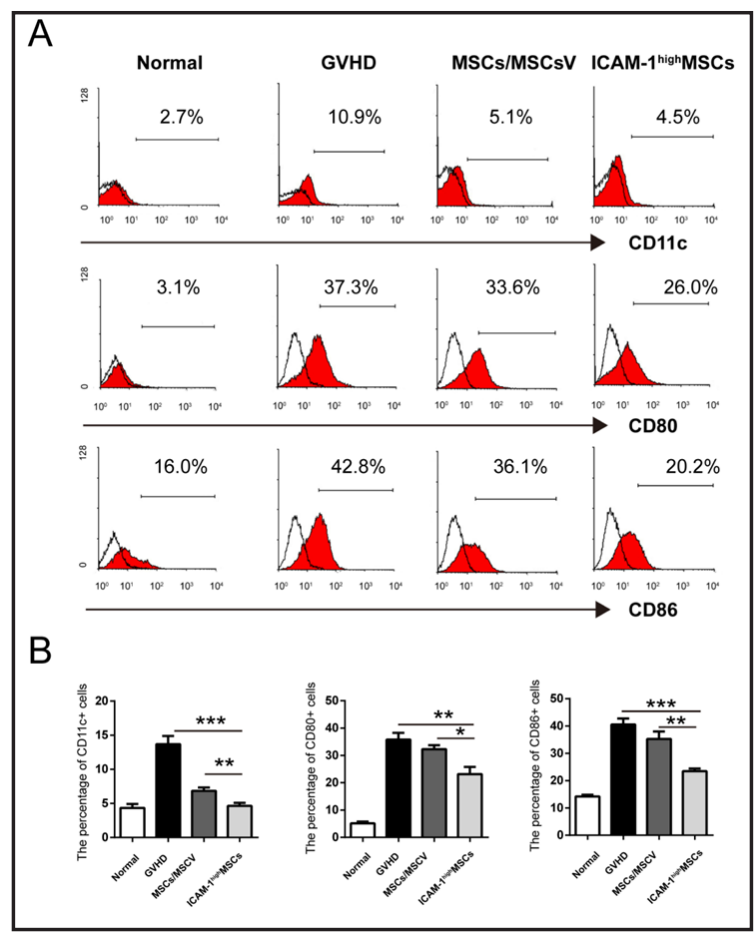
(Fig. 3A). The lowest GVHD scores were detected with the infusion of $5 \times 10^{5}$ ICAM1 MSCs (Fig. 3B). Consistent with the prolonged survival time, the GVHD + ICAM$1^{\mathrm{HIGH}}$ MSCS group of mice also had significantly alleviated clinical histological scores compared with the GVHD and GVHD + MSCs groups. A histological examination at the end of the experiment demonstrated that an ICAM- $1^{\mathrm{HIGH}}$ MSCS infusion dramatically decreased lymphocyte infiltration in the GVHD target tissues, including the liver, intestine and lung (Fig. 3C).

\section{Enhanced ICAM-

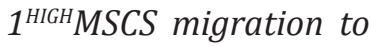 SLOs in a GVHD mouse model}

We further examined whether MSCs could migrate to target organs and SLOs in vivo. Forty-eight hours after the injection of $5 \times 10^{5}$ cells, there were

Fig. 6. ICAM- $1^{\text {high }}$ MSCs regulated the differentiation of $\mathrm{T}$ cells in vivo. (A) The levels of the intracellular cytokines of donor $\mathrm{T}$ cells were detected by fluorescenceactivated cell sorting (FACS) analysis at day 7 after transfusion. Gated on $\mathrm{H} 2 \mathrm{~Kb}$, the frequencies of Th1 cells (IFN- $\gamma$ producing $\mathrm{CD} 4+$ cells) are shown.

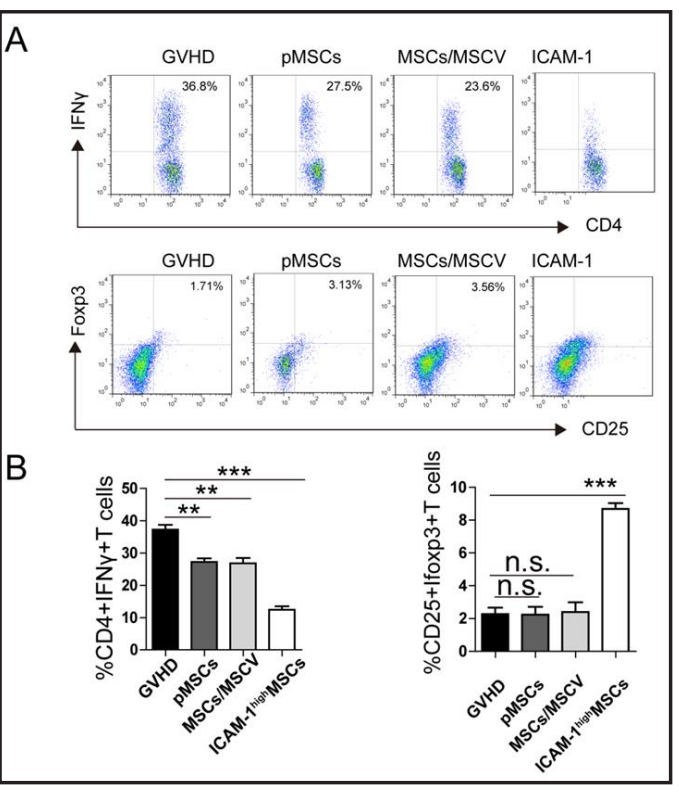
In addition, the frequencies of Treg cells are also detected. Gated on CD4, the frequencies of Treg cells (CD25+ Foxp3+ cells) are shown. (B) The difference in percentage of Th1 and Treg cells was analyzed. The data are the mean of 5 independent experiments and a representative result is shown. The data represent the mean value $\pm \mathrm{SD}$ of at least 5 mice $\quad{ }^{*}, \mathrm{p}<0.05$; **, $\mathrm{p}<0.01 ;{ }^{* * *}, \mathrm{p}<0.001$ compared with the MSCs or MSC/MSCV infusion group). Abbreviations: GVHD, graft-versus-host disease; pMSCs, primary MSCs; MSCs/MSCV, a murine MSC line C3H10T1/2 cells transfected with the empty vector; ICAM- $1^{\text {high }}$ MSCs, overexpressing-ICAM-1 MSC cell line.

\section{KARGER}


almost an undetectable amount of MSCs in the liver and intestine of mice. $\mathrm{CFSE}^{+}$MSCs and $\mathrm{CFSE}^{+} \mathrm{MSCs} / \mathrm{MSCV}$ appeared sporadically in the spleen and lymph nodes. In contrast, a significantly larger number of $\mathrm{CFSE}^{+}$ICAM- $1^{\text {high }}$ MSCs was dispersed in SLOs in model mice $(p<0.05$; Fig. 4).

Overexpressing ICAM-1 enhanced the immunosuppression capacity of MSCs for DCs in vivo

Since ICAM-1 MSCs gained an immunosuppression capacity for DCs in vitro, we further examined whether they could suppress DCs function in the spleen after intravenous infusion. Seven days after infusion, surface expression of CD11c was slightly decreased in the ICAM- $1^{\text {high }}$ MSCs group compared with MSCs/MSCV $(p=0.035)$. Expression of CD80 and CD86 also had an obvious reduction in the ICAM- $1^{\mathrm{HIGH}}$ MSCS group compared with MSCs/MSCV ( $p=0.006$ and $p=0.003$; Fig. 5).

Enhanced expression of ICAM-1 induced the induction of Treg cells and reduced the percentage of Th1 cells

In GVHD pathophysiology, the recruitment of donor-derived T cells to the tissue is an early and key event [1] [1]. GVHD is a result of naive T cell responses. Owing to their ability to differentiate into various effector $\mathrm{T}$ cell subsets - namely, Th1 cells, Th2 cells and Th17 cells - CD4+T cells are particularly important in the initiation of GVHD. At day 7 after BMT, PMA/iono stimulation of spleen cells showed that GVHD-associated Th1 cells strongly increased in mice developing GVHD. Importantly, co-transplantation of ICAM- $1^{\text {high }}$ MSCs significantly decreased the percentage of Th1 cells (Fig. 6). Treg are defined by their expression of CD4, CD25 and the transcription factor forkhead box P3 (FOXP3), and control innate and adaptive immune responses [22]. Among these group, Treg cell skewing in ICAM-1MSC-treated mice was also observed (Fig. 6).

\section{Enhanced expression of ICAM-1 affected the expression of T cell related cytokine}

Th1 type cytokine, such as IFN- $\gamma$ and TNF $\alpha$ have been shown to be aetiological factors in the induction of GVHD [23]. Th2-type cytokines, such as IL-4, can reduce acute GVHD [24]. The mRNA levels of the Th1 cytokines IFN- $\gamma$ and TNF $\alpha$ were strongly decreased in mice co-

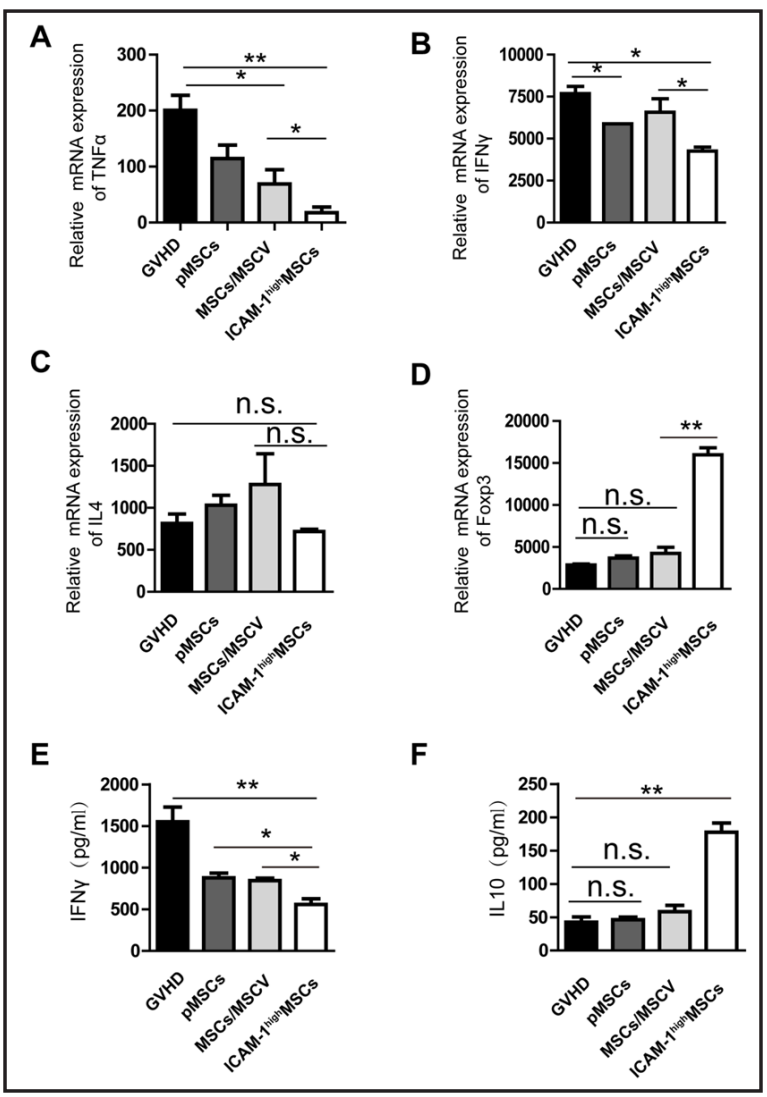

Fig. 7. ICAM-1 $1^{\text {high }}$ MSCs decreased Th1-ype cytokines and increased Treg-type cytokines. Seven days after MSC infusion, mice were euthanized and splenic cells were isolated from recipients. Then, q-PCR was performed to assess the expression of TNF $\alpha$, IFN- $\gamma$ and IL- 4 , and the expression relative to Hprt was calculated (A, B, C, and D). The IFN- $\gamma$ and IL-10 concentrations were determined in the serum of transplanted animals 7 days after MSC infusion, according to the reagent protocols of the quantitative determination kit (E, F). The data represent the mean value \pm SD of at least 5 mice. The data are representative of three independent experiments . $\left({ }^{*}, \mathrm{p}<0.05\right.$; ${ }^{* *}, \mathrm{p}<0.01$; ***, $\mathrm{p}<0.001$, compared with the pMSCs or MSC/MSCV infusion group). Abbreviations: TNF- $\alpha$, tumour necrosis factor $\alpha$; IFN- $\gamma$, interferon $\gamma$; IL-4, interleukin-4; Foxp3, forkhead box P3; IL-10, interleukin-10. 
transplanted with ICAM-1 MSCs (Fig. 7A and 7B), while the expression of IL4 displayed no obvious change among each group (Fig. 7C). Most importantly, Treg cell skewing in ICAM1MSC-treated mice was also observed, and mRNA expression of foxp3 was also up-regulated (Fig. 7D). Th1 serum cytokine concentration of IFN- $\gamma$ obviously decreased in the group treated with ICAM-1 MSCs (Fig. 7E), while Treg related serum cytokine concentration of IL10 was elevated in mice treated with ICAM-1 $1^{\text {high }}$ MSCs compared to GVHD group (Fig. 7F).

\section{Discussion}

GVHD presents a major complication after allogeneic bone marrow transplantation. Idiopathic pneumonia syndrome (IPS) may be considered as a pulmonary manifestation of GVHD. The immunosuppressive properties of MSCs have been established, and the treatment of GVHD/IPS with MSCs has been reported, which suggests that MSCs can inhibit undesirable immune responses [25,26]. Recently, several clinical trials using MSCs infusions have been reported. However, these reports were confusing, as they did not show any benefit after MSCs infusion [27]. The experimental basis of MSCs is somewhat limiting the clinical use of MSCs. MSCs do not readily migrate into the appropriate target tissues, and this creates a major obstacle for the use of MSCs.

Some methods have been developed to enhance the migration of MSCs to target organs. Attaching a synthetic high affinity peptidomimetic ligand (LLP2A) to integrin $\alpha 4 \beta 1$ on the MSCs surface can direct MSCs to the designated sites [28]. The high expression of CXCR4 enhanced the homing and engraftment of MSCs by increasing the response to SDF-1 [29, 30]. Additionally, CCR7 overexpression enhanced the homing of MSCs to secondary lymphoid organs and thus highly intensified their in vivo immunomodulatory effect [31].

To our knowledge, we show for the first time, that the positive therapeutic effects of ICAM1 MSCs in GVHD animal models with improved survival, low GVHD scores and attenuated tissue injury. Of note, transplanted ICAM-1 ${ }^{\text {high }}$ MSCs enhanced their homing to SLOs in vivo. SLOs are also a strategically positioned collecting station for initiating the adaptive immune response, where a naive $\mathrm{T}$ cell scan the surfaces of antigen-presenting cells for cognate antigens and activates and generates armed effectors under the signaling cues from the APC [32-35]. And it was also found that in the absence of secondary stimulation even ${ }^{\text {high }}$ numbers of host-reactive T cells are hardly activated in GVHD [36]. DCs, one kind of professional APC, are the most potent initiator of in vivo immune responses. Overexpressing ICAM-1 enhanced the immunosuppression capacity of MSCs for DCs and T cells in vitro. Whether the increased quantity of ICAM- $1^{\text {high }}$ MSCs counters DCs and T cells in SLOs, it can result in enhanced immunosuppression capacity for DCs and T cells. Our results here demonstrate that the presence of ICAM- $1^{\text {high }}$ MSCs can significantly inhibit maturation and differentiation of DCs, and regulated CD4+Th cell differentiation in vivo. Therefore, the results indicated that ICAM-1 can alter the migratory capacity of MSCs to SLOs and thus, show the enhanced immunosuppression capacity to effector cells.

The fate of the murine GVHD model is determined at least partially by the types and numbers of effector T cells. It is generally understood that Th1 cells and pro-inflammatory molecules such as IFN- $\gamma$ and TNF $\alpha$ have been shown to be aetiological factors in the induction of GVHD $[37,38]$. These pro-inflammatory molecules contribute to a systemic syndrome with variable presentations of weight loss, diarrhea and skin changes, and increased mortality. ICAM- ${ }^{\text {high }}$ MSCs infusion reduced the percentage of Th1, and the expression of IFN- $\gamma$ and TNF $\alpha$. The presence of Treg cells was recognized as one of the critical factors in attenuating GVHD. Natural Treg cells, which are defined by their expression of CD4, CD25 and FOXP3, regulate innate and adaptive immune response $[39,40]$. Treg cell impairment is associated with a loss of tolerance, as well as with GVHD [41]. So our observations suggest that the interruption of ICAM-1 $1^{\text {high }}$ MSCs may affect the balance of regulatory and effector T cells in SLOs. 
Several papers have shown that MSCs suppress T-cell immunity in vitro by both direct cell-cell contact and the soluble substances that MSCs release in the supernatants [42-44]. Our results here showed that ICAM-1 MSCs could suppress DC differentiation to maDCs, and suppress the T cell immune response as shown by the MLR and LTT tests in vitro. Additionally, when DCs were co-cultured with MSCs-conditioned medium in vitro, we observed that the ICAM-1 ${ }^{\text {high }}$ MSCs supernatant had a greater inhibition efficiency for DCs compared with other groups (unpublished data), which clearly indicates that such an effect not only depends on the physical contact between MSCs and DCs, but also on soluble factors released by ICAM1 MSCs. These soluble factors maybe not constitutively secreted by MSCs. It is important to further explore which molecules secreted by ICAM-1 MSCs plays a crucial role in immune regulation.

\section{Conclusion}

Taken together, our results indicate that ICAM- $1^{\text {high } M S C s}$ had greater migration activity to the modulatory center (SLOs) of immune responses in a mouse model. Given that MSCs express a low level of ICAM-1, it is plausible to speculate that a novel strategy could be applied to human MSCs to achieve targeted organ trafficking to attenuate GVHD.

\section{Acknowledgements}

This work was supported by the National Key and Development Program of China (grant number 2016YFC1000305) and the Origin cell Technology Group grant.

\section{Disclosure Statement}

The authors have no competing interests.

\section{References}

$>1$ Johnston L: Acute graft-versus-host disease: differing risk with differing graft sources and conditioning intensity. Best Pract Res Clin Haematol 2008;21:177-192.

2 Shlomchik WD, Couzens MS, Tang CB, McNiff J, Robert ME, Liu J, Shlomchik MJ, Emerson SG: Prevention of graft versus host disease by inactivation of host antigen-presenting cells. Science 1999;285:412-415.

3 Anderson BE, McNiff JM, Jain D, Blazar BR, Shlomchik WD, Shlomchik MJ: Distinct roles for donor- and hostderived antigen-presenting cells and costimulatory molecules in murine chronic graft-versus-host disease: requirements depend on target organ. Blood 2005;105:2227-2234.

-4 Matte CC, Liu J, Cormier J, Anderson BE, Athanasiadis I, Jain D, McNiff J, Shlomchik WD: Donor APCs are required for maximal GVHD but not for GVL. Nat Med 2004;10:987-992.

-5 Aggarwal S, Pittenger MF: Human mesenchymal stem cells modulate allogeneic immune cell responses. Blood 2005; 105:1815-1822.

-6 Pittenger MF, Mackay AM, Beck SC, Jaiswal RK, Douglas R, Mosca JD, Moorman MA, Simonetti DW, Craig S, Marshak DR: Multilineage potential of adult human mesenchymal stem cells. Science 1999;284:143-147.

7 Hashmi S, Ahmed M, Murad MH, Litzow MR, Adams RH, Ball LM, Prasad VK, Kebriaei P, Ringden O: Survival after mesenchymal stromal cell therapy in steroid-refractory acute graft-versus-host disease: systematic review and meta-analysis. Lancet Haematol 2016;3: 45-52.

-8 Sudres M, Norol F, Trenado A, Gregoire S, Charlotte F, Levacher B, Lataillade JJ, Bourin P, Holy X, Vernant JP, Klatzmann D, Cohen JL: Bone marrow mesenchymal stem cells suppress lymphocyte proliferation in vitro but fail to prevent graft-versus-host disease in mice. J Immunol 2006;176:7761-7767. 


\section{Cellular Physiology Cell Physiol Biochem 2018;46:2624-2635 \begin{tabular}{l|l|} 
and Biochemistry Published 10.1159/000489689 & $\begin{array}{l}\text { C } 2018 \text { The Author(s). Published by S. Karger AG, Basel } \\
\text { www.karger.com/cpb }\end{array}$ \\
\hline
\end{tabular}}

Tang et al.: ICAM-1highMSCs on aGVHD

9 Tolar J, Le Blanc K, Keating A, Blazar BR: Concise review: hitting the right spot with mesenchymal stromal cells. Stem Cells 2010;28:1446-1455.

10 Kebriaei P, Robinson S: Treatment of graft-versus-host-disease with mesenchymal stromal cells. Cytotherapy 2011;13:262-268.

11 Kang SK, Shin IS, Ko MS, Jo JY, Ra JC: Journey of mesenchymal stem cells for homing: strategies to enhance efficacy and safety of stem cell therapy. Stem Cells Int 2012;2012:342968.

-12 Karp JM, Leng Teo GS: Mesenchymal stem cell homing: the devil is in the details. Cell Stem Cell 2009;4:206216.

13 Pribila JT, Quale AC, Mueller KL, Shimizu Y: Integrins and T cell-mediated immunity. Annu Rev Immunol 2004;22:157-180.

14 Isobe M, Suzuki J, Yamazaki S, Yazaki Y, Horie S, Okubo Y, Maemura K, Yazaki Y, Sekiguchi M: Regulation by differential development of Th1 and Th2 cells in peripheral tolerance to cardiac allograft induced by blocking ICAM-1/LFA-1 adhesion. Circulation 1997;96:2247-2253.

15 Sumagin R, Brazil JC, Nava P, Nishio H, Alam A, Luissint AC, Weber DA, Neish AS, Nusrat A, Parkos CA: Neutrophil interactions with epithelial-expressed ICAM-1 enhances intestinal mucosal wound healing. Mucosal Immunol 2016;9:1151-1162.

16 Shi Y, Su J, Roberts AI, Shou P, Rabson AB, Ren G: How mesenchymal stem cells interact with tissue immune responses. Trends Immunol 2012;33:136-143.

17 Ren G, Roberts AI, Shi Y: Adhesion molecules: key players in Mesenchymal stem cell-mediated immunosuppression. Cell Adh Migr 2011;5:20-22.

18 Ren G, Zhao X, Zhang L, Zhang J, L'Huillier A, Ling W, Roberts AI, Le AD, Shi S, Shao C, Shi Y: Inflammatory cytokine-induced intercellular adhesion molecule- 1 and vascular cell adhesion molecule-1 in mesenchymal stem cells are critical for immunosuppression. J Immunol 2010;184:2321-2328.

19 Xu FF, Zhu H, Li XM, Yang F, Chen JD, Tang B, Sun HG, Chu YN, Zheng RX, Liu YL, Wang LS, Zhang Y: Intercellular adhesion molecule-1 inhibits osteogenic differentiation of mesenchymal stem cells and impairs bio-scaffold-mediated bone regeneration in vivo. Tissue Eng Part A 2014;20:2768-2782.

20 Kaplan DH, Anderson BE, McNiff JM, Jain D, Shlomchik MJ, Shlomchik WD: Target antigens determine graftversus-host disease phenotype. J Immunol 2004;173:5467-5475.

21 Mellman I, Steinman RM: Dendritic cells: specialized and regulated antigen processing machines. Cell 2001;106:255-258.

22 Piccirillo CA, Shevach EM: Naturally-occurring CD4+CD25+ immunoregulatory T cells: central players in the arena of peripheral tolerance. Semin Immunol 2004;16:81-88.

23 Cooke KR, Hill GR, Crawford JM, Bungard D, Brinson YS, Delmonte J Jr, Ferrara JL: Tumor necrosis factoralpha production to lipopolysaccharide stimulation by donor cells predicts the severity of experimental acute graft-versus-host disease. J Clin Invest 1998;102:1882-1891.

24 Leveson-Gower DB, Olson JA, Sega EI, Luong RH, Baker J, Zeiser R, Negrin RS: Low doses of natural killer $\mathrm{T}$ cells provide protection from acute graft-versus-host disease via an IL-4-dependent mechanism. Blood 2011;117:3220-3229.

25 Polchert D, Sobinsky J, Douglas G, Kidd M, Moadsiri A, Reina E, Genrich K, Mehrotra S, Setty S, Smith B, Bartholomew A: IFN-gamma activation of mesenchymal stem cells for treatment and prevention of graft versus host disease. Eur J Immunol 2008;38:1745-1755

-26 Qiao SK, Ren HY, Shi YJ, Liu W: Allogeneic Compact Bone-Derived Mesenchymal Stem Cell Transplantation Attenuates the Severity of Idiopathic Pneumonia Syndrome in a Murine Bone Marrow Transplantation Model. Cell Physiol Biochem 2016;40:1656-1669.

27 Sudres M, Norol F, Trenado A, Gregoire S, Charlotte F, Levacher B, Lataillade JJ, Bourin P, Holy X, Vernant JP, Klatzmann D, Cohen JL: Bone marrow mesenchymal stem cells suppress lymphocyte proliferation in vitro but fail to prevent graft-versus-host disease in mice. J Immunol 2006;176:7761-7767.

28 Guan M, Yao W, Liu R, Lam KS, Nolta J, Jia J, Panganiban B, Meng L, Zhou P, Shahnazari M, Ritchie RO, Lane NE: Directing mesenchymal stem cells to bone to augment bone formation and increase bone mass. Nat Med 2012;18:456-462.

29 Liu HB, Liu SB, Li Y, Wang XH, Xue WJ, Ge GQ Luo XH: The Role of SDF-1-CXCR4/CXCR7 Axis in the Therapeutic Effects of Hypoxia-Preconditioned Mesenchymal Stem Cells for Renal Ischemia/Reperfusion Injury. PloS One 2012;7: e34608. 


\section{Cellular Physiology Cell Physiol Biochem 2018;46:2624-2635 \begin{tabular}{l|l|l} 
DOI: 10.1159/000489689 & $\begin{array}{l}\text { O } 2018 \text { The Author(s). Published by S. Karger AG, Basel } \\
\text { www.karger.com/cpb }\end{array}$
\end{tabular}}

Tang et al.: ICAM-1highMSCs on aGVHD

-30 Yang DZ, Sun SJ, Wang ZG, Zhu PF, Yang ZL, Zhang B: Stromal Cell-Derived Factor-1 Receptor CXCR4Overexpressing Bone Marrow Mesenchymal Stem Cells Accelerate Wound Healing by Migrating into Skin Injury Areas. Cell Reprogram 2013;15:206-215.

-31 Li H, Jiang Y, Jiang X, Guo X, Ning H, Li Y, Liao L, Yao H, Wang X, Liu Y, Zhang Y, Chen H, Mao N: CCR7 Guides Migration of Mesenchymal Stem Cell to Secondary Lymphoid Organs: A Novel Approach to Separate GvHD from GvL Effect. Stem Cells 2014;32:1890-1903.

-32 Stoll S, Delon J, Brotz TM, Germain RN: Dynamic imaging of T cell-dendritic cell interactions in lymph nodes. Science 2002;296:1873-1876.

-33 Malhotra D, Fletcher AL, Turley SJ: Stromal and hematopoietic cells in secondary lymphoid organs: partners in immunity. Immunol Rev 2013;251:160-176.

-34 Miller MJ, Wei SH, Parker I, Cahalan MD: Two-photon imaging of lymphocyte motility and antigen response in intact lymph node. Science 2002;296:1869-1873.

35 Mempel TR, Henrickson SE, von Andrian UH: T-cell priming by dendritic cells in lymph nodes occurs in three distinct phases. Nature 2004;427:154-159.

-36 Gassa A, Kalkavan H, Jian F, Duhan V, Khairnar V, Shaabani N, Honke N, Carpinteiro A, Botezatu L, Crivello P, Dolff S, Ferencik S, Häussinger D: High Frequencies of Anti-Host Reactive CD8(+) T Cells Ignore NonHematopoietic Antigen after Bone Marrow Transplantation in a Murine Model. Cell Physiol Biochem 2016;38:1343-1353.

-37 Nestel FP, Price KS, Seemayer TA, Lapp WS: Macrophage priming and lipopolysaccharide-triggered release of tumor necrosis factor alpha during graft-versus-host disease. J Exp Med 1992;175:405-413.

-38 Lu Y, Waller EK: Dichotomous role of interferon-gamma in allogeneic bone marrow transplant. Biol Blood Marrow Transplant 2009;15:1347-1353.

-39 Serra P, Amrani A, Yamanouchi J, Han B, Thiessen S, Utsugi T, Verdaguer J, Santamaria P: CD40 ligation releases immature dendritic cells from the control of regulatory CD4+CD25+ T cells. Immunity 2003;19:877-889.

40 Janssens W, Carlier V, Wu B, VanderElst L, Jacquemin MG, Saint-Remy JM: CD4+CD25+ T cells lyse antigenpresenting B cells by Fas-Fas ligand interaction in an epitope-specific manner. J Immunol 2003;171:46044612.

41 Buckner JH: Mechanisms of impaired regulation by CD4(+)CD25(+)FOXP3(+) regulatory T cells in human autoimmune diseases. Nat Rev Immunol 2010;10:849-859.

42 Krampera M, Glennie S, Dyson J, Scott D, Laylor R, Simpson E, Dazzi F: Bone marrow mesenchymal stem cells inhibit the response of naive and memory antigen-specific T cells to their cognate peptide. Blood 2003;101:3722-3729.

43 Di Nicola M, Carlo-Stella C, Magni M, Milanesi M, Longoni PD, Matteucci P, Grisanti S, Gianni AM: Human bone marrow stromal cells suppress T-lymphocyte proliferation induced by cellular or nonspecific mitogenic stimuli. Blood 2002;99:3838-3843.

44 Li H, Guo Z, Jiang X, Zhu H, Li X, Mao N: Mesenchymal stem cells alter migratory property of T and dendritic cells to delay the development of murine lethal acute graft-versus-host disease. Stem Cells 2008;26:25312541. 